\title{
Factores de Deserción Estudiantil en Ingeniería: Una Aplicación de Modelos de Duración
}

\author{
Christian J. Díaz \\ Universidad Católica de la Santísima Concepción, Facultad de Ingeniería, Alonso de Ribera 2850, \\ Concepción-Chile (e-mail: chdiaz@ucsc.cl)
}

\begin{abstract}
Resumen
Se analizó la deserción estudiantil universitaria, en estudiantes de carreras de ingeniería de la cohorte del año 2004 hasta enero del año 2007. Se evaluó la retención utilizando un modelo de Kaplan-Meier, y se determinaron factores de la deserción con un modelo de riesgos proporcionales de Cox. Los resultados muestran que la mayor probabilidad de desertar se presenta en los tres primeros semestres, y en estudiantes que provienen de colegios financiados por el estado. Son factores determinantes de la retención un alto promedio ponderado acumulado, disponer de crédito universitario, a mayor puntaje de la prueba de selección universitaria, provenir de un establecimiento educacional de enseñanza media científico-humanista y altos ingresos promedio familiar. Se analiza también los factores de deserción más recurrentes y se proponen medidas para disminuir la deserción estudiantil universitaria.
\end{abstract}

Palabras clave: deserción estudiantil, retención estudiantil, modelo de Cox, riesgo de deserción

\section{Student Dropout Factors in Engineering: An Application of Duration Models}

\begin{abstract}
College drop-out of undergraduate engineering students from 2004 until January 2007 was analysed using the Kaplan-Meier model to evaluate the retention rate of students. Drop-out factors were determined using a Cox model for proportional risks. The results show that it is most likely that dropouts take place within the first three terms and mainly affect those students coming from public school, financed by the government. Retention decisive factors are: high average high school grades, access to the national system of university credit, high average grade in the national university selection exam, secondary education with a scientific and humanities oriented curriculum, and high family incomes. The most common drop-out factors are also discussed and solutions to diminish university desertion are proposed.
\end{abstract}

Keywords: Cox model, dropout risk, student dropout, student retention, survival analysis 


\section{INTRODUCCIÓN}

La deserción, permanencia y titulación de los estudiantes universitarios se ha transformado en un elemento estratégico en las instituciones en términos de rendición de cuentas, y también para las recientes iniciativas del Ministerio de Educación de Chile, al incorporar indicadores de eficiencia docente tales como, la tasa de retención y de titulación a través de la Ley № 19.986 que establece una estructura de crédito para financiar los estudios universitarios. Este fondo de financiamiento estudiantil, a partir del año 2006, se distribuye utilizando un modelo que fija un arancel de referencia para cada carrera de universidades pertenecientes al Consejo de Rectores de Universidades Chilenas (CRUCH). A lo anterior se suma, la Ley $\mathrm{N}^{\circ} 20.027$ que incorpora la deserción estudiantil como indicador de evaluación de las instituciones de educación superior.

De acuerdo con la legislación actual en Chile, la educación formal está constituida por niveles que aseguran la unidad del proceso educativo y facilitan la continuidad del mismo a lo largo de la vida de las personas. Estos niveles que pueden ser con financiamiento privado, del Estado o compartidos son: la enseñanza básica, la enseñanza media, y la educación superior. Es así como el Estado reconoce las siguientes instituciones de educación superior: universidades, institutos profesionales, centros de formación técnica y establecimientos de las fuerzas armadas y de orden (Ley 18.962, 1990).

De acuerdo al Ministerio de Educación de Chile (2007), el número total de universidades del país sumaban 61, de las cuales 25 pertenecen al $\mathrm{CRUCH}, 16$ son estatales y 9 particulares de carácter público, y 36 son privadas que no reciben Aporte Fiscal Directo (AFD). El AFD es el instrumento de financiamiento del Estado para las universidades del $\mathrm{CRUCH}$. Consiste en un subsidio de libre disponibilidad asignado en un 95\% conforme criterios históricos y el 5\% restante de acuerdo con indicadores de eficiencia anuales. Otro aporte dirigido a todas las instituciones de educación superior (universidades, institutos profesionales y centros de formación técnica) es el Aporte Fiscal Indirecto (AFI). Este es un aporte concursable, para los mejores 27.500 puntajes en la prueba de selección, distribuidos a través de la matrícula de primer año.

Desde el año 2003, las universidades que componen el CRUCH emplean nuevas pruebas con puntajes mínimos para seleccionar a los estudiantes que ingresan a sus carreras. Éstas constituyen cuatro pruebas llamadas Pruebas de Selección Universitaria (PSU), las que son desarrolladas, administradas, aplicadas y reportadas por el Departamento de Evaluación, Medición y Registro Educacional de la Universidad de Chile (DEMRE). Las PSU son instrumentos de evaluación educacional que miden la capacidad de razonamiento de los postulantes egresados de la enseñanza media, teniendo como medio, los contenidos del plan de formación general de Lenguaje y Comunicación, de Matemática, de Historia y Ciencias Sociales y de Ciencias. Esta última incluye a biología, física y química. Los estudiantes deben rendir en forma obligatoria Lenguaje y Comunicación, y Matemática, y elegir entre Historia y Ciencias Sociales y Ciencias. Sin embargo, si lo estiman, pueden optar por las cuatro pruebas. En cuanto a las universidades privadas no pertenecientes al $\mathrm{CRUCH}$, éstas no exigen un puntaje mínimo para ingresar a las carreras profesionales ofrecidas.

En Chile, de acuerdo a estimaciones basadas en estadísticas nacionales, la tasa de deserción global de pregrado, evaluada para el año 2004 , es cercana al $53,7 \%$. Las áreas del conocimiento más críticas son Humanidades y Derecho con cifras del orden de $80 \%$ de deserción, y las más eficientes son las áreas de Educación y Salud con un $37 \%$ y un $27 \%$, respectivamente. Por género, las mujeres poseen una tasa de deserción promedio más baja que los varones de $43 \%$ y $50 \%$, respectivamente (González, 2005). Particularmente, González (2005) basado en el seguimiento de desertores de cuatro universidades, mostró que la tasa de deserción en Ingeniería es de alrededor de un 26\%. Sin embargo, este valor puede ser mayor en otras universidades.

Para el sistema nacional el efecto de la deserción, en términos de costos, es de 96,2 millones de dólares. De acuerdo a esta cifra, el gasto por abandono es equivalente al $26 \%$ del gasto público en educación universitaria, mientras que representa el 10,2\% del gasto total (público y privado) en el sector universitario (UNESCO, 2004). 
En este contexto, la Facultad de Ingeniería de la Universidad Católica de Santísima Concepción, no ha estado ajena al problema de deserción, por lo cual se ha propuesto analizar este problema a través de distintas cohortes de estudiantes. Debido al carácter privado de la información específica evaluada, se ha mantenido la reserva de los nombres de las carreras investigadas.

Considerando la escasa investigación nacional referida a la modelación cuantitativa del fenómeno de la deserción, a fin de diseñar acciones que propendan a la disminución de la misma, el presente estudio tiene como propósito estimar un modelo que explique la deserción estudiantil universitaria de pregrado e identifique los factores que la determinan.

\section{DESARROLLO}

\section{La deserción universitaria en Chile}

En Chile la deserción universitaria no ha sido ampliamente investigada ni se cuenta con una base estadística por instituciones a nivel nacional; sin embargo, el Ministerio de Educación ha mostrado preocupación por los efectos del fenómeno de la deserción. En una reciente investigación, dirigida por el Consejo Superior de Educación (CSE) sobre la base de la información recopilada desde Indicadores, Números y Datos sobre Instituciones y Carreras de Educación Superior (INDICES, 2006), se afirma que las universidades del $\mathrm{CRUCH}$ tienen mayor retención de su alumnado, manteniendo al $73 \%$ de ellos al finalizar el segundo año, a diferencia de las instituciones privadas que mantienen sólo al $65 \%$. Otra conclusión del estudio, revela que los alumnos provenientes de colegios financiados por el estado conforman la mayoría del total que ingresa a la educación superior, y son éstos los que presentan los mayores indicadores de deserción en todo el sistema de educación terciaria.

En el país, son escasos los estudios que han intentado modelar e identificar las causas por las cuales un estudiante decide abandonar sus estudios superiores, y con esto formular políticas y mecanismos de reingeniería educativa que permitan la permanencia de los mismos dentro del sistema de educación superior o de la institución universitaria (González, 2005; Donoso y Schiefelbein, 2007; Canales y De los Ríos, 2007). Lo que prevalece en estos estudios, es la caracterización de la población desertora, la construcción de indicadores de eficiencia y efectividad del proceso formativo y la propia estadística descriptiva del fenómeno a través de las distintas cohortes.

Los estudios, excepto el de González y Uribe (2002) y González (2005), son análisis de casos de instituciones y el tratamiento metodológico ha sido de tipo estático (Prieto, 2002), sin considerar la evolución del fenómeno en el tiempo. Otro problema que enfrentan los estudios, en países como Chile, es la calidad de los datos en cuanto a confiabilidad y oportunidad. Cuando se tienen datos de baja confiabilidad no es posible efectuar una comparación (Ishitani y Zinder, 2006).

\section{La deserción desde la perspectiva institucional}

Actualmente, a pesar de que la definición de deserción estudiantil está en discusión, existe consenso en definirla como un abandono voluntario que puede ser explicado por diferentes categorías de variables: socioeconómicas, individuales, institucionales y académicas. Sin embargo, la forma de operacionalizarlas depende del punto de vista desde el cual se haga el análisis, esto es: individual, institucional y estatal o nacional. Tinto (1989), afirma que el estudio de la deserción en la educación superior es extremadamente complejo, ya que implica no sólo una variedad de perspectivas, sino que además, una gama de diferentes tipos de abandono.

Desde el punto de vista institucional, todos los estudiantes que abandonan su educación superior pueden ser clasificados como desertores. Varios autores asocian la deserción con los fenómenos de "mortalidad" académica y retiro forzoso, en este sentido, cada estudiante que abandona la institución crea un lugar vacante que pudo ser ocupado por otro alumno que permaneciera en sus estudios, por lo cual, la pérdida de estudiantes causa serios problemas financieros a las instituciones al producir inestabilidad en la fuente de sus ingresos (Tinto, 1989). 
Si bien el presente estudio presenta un enfoque institucional para el análisis de la deserción, Bourdieu (1984) señala que los estudiantes que ingresan a la educación superior, pertenecen a clases sociales privilegiadas de la sociedad y que han llegado a este nivel, no por ser más inteligentes que los estudiantes pobres y pertenecientes a clases desfavorecidas, sino porque han recibido un capital cultural constituido en informaciones y conocimientos que no poseen los demás. Desde la teoría social, Boudon (1983) propone el individualismo metodológico, presentando una analogía entre el "ser individual" y el "ser colectivo". Si el ser humano individual está dotado de un "instinto de conservación" que le impide natural y espontáneamente obrar en contra de sí mismo, de la misma manera el "ser colectivo" tratará de realizar todo aquello que lo favorezca, y se abstendrá de llevar a cabo acciones que vayan en detrimento de la colectividad a la cual pertenece. Esta concepción sociológica, es relevante al analizar la capacidad de adaptación de las redes sociales que poseen los estudiantes al ingresar a la universidad, y cómo se generan los nuevos espacios de convivencia con la comunidad universitaria

\section{Síntesis de modelos teóricos de análisis para la deserción estudiantil}

Las investigaciones recientes han generado distintas aproximaciones teóricas al fenómeno de la deserción estudiantil. Los modelos psicológicos señalan que la decisión de desertar es afectada por conductas previas al ingreso a la universidad, al igual que la motivación al logro y el compromiso con metas académicas (Bean y Eaton, 2001).

Los modelos sociológicos enfatizan respecto a la influencia en la deserción de factores externos al individuo, adicionales a los psicológicos (Spady, 1971). Este autor encontró seis predictores de la deserción estudiantil en los dos primeros años de estudio universitario norteamericano: integración académica, integración social, estado socioeconómico, género, calidad de la carrera y el promedio de notas en cada semestre.

Los modelos económicos explican el problema mediante dos enfoques. La primera, según la relación costo/beneficio (beneficios sociales y económicos asociados a los estudiantes), donde el estudiante percibe esta relación como menor que relaciones que involucren actividades alternas (por ejemplo un trabajo), por lo que el estudiante opta por permanecer en la universidad. La segunda, es la focalización de subsidio que consiste en la entrega de subsidios como una forma de influir sobre la disminución de la deserción (Cabrera et al., 1993; St. John et al., 2000).

Otro es el modelo organizacional, el que indica que la deserción depende de las capacidades de la organización en la integración social, y principalmente en el abandono de los estudiantes que ingresan a ella (Kuh, 2002). Así mismo, Tinto (1975), basado en la teoría Spady (1971) elaboró el Modelo Integración Estudiantil, considerado como un modelo paradigmático del estudio de la deserción estudiantil. En éste, interactúan características preuniversitarias que influyen directamente en el compromiso inicial con la institución y sus metas académicas. El estudiante que ingresa, evalúa permanentemente su integración académica y social en la institución. A mayor grado de integración social y académica, mayor compromiso institucional, lo que permite la disminución de las tasas de deserción.

El modelo de Bean (1985), conocido como el Modelo de Deserción de Estudiantes, se basa en los resultados de las investigaciones de Tinto (1975) incorporando a éste las características del modelo de productividad, desarrollado en el contexto de las organizaciones laborales. Reemplaza las variables del ambiente laboral por las más adecuadas al entorno de la educación superior, de tal forma que la satisfacción con los estudios es similar a la satisfacción con el trabajo. Bean (1985), encuentra que los factores institucionales afectan la satisfacción del estudiante con la universidad y por ende la intensión de desertar.

Varios estudios han probado los modelos de Tinto (1975) y de Bean (1985). En este contexto, Cabrera et al. (1993), indican que los dos modelos anteriores son complementarios, presentando posteriormente un modelo integrado, concluyendo que la especificación estructural de los procesos psicológicos y sociológicos, subyacen en la conducta del abandono. Si bien estos estudios han 
demostrado que ayudan a describir el comportamiento natural de los estudiantes, carecen de una mayor aplicación práctica (Díaz, 2008).

\section{Factores que afectan a la deserción estudiantil}

Una serie de factores se consideran para explicar la deserción estudiantil universitaria, como: género, raza, tipo de colegio secundario, desempeño académico, ingreso económico entre otros (Pascarella y Chapman, 1983; Braxton et al. 1988). Pascarella y Terenzini (1983), encontraron una relación positiva entre el nivel educacional de los padres y las tasas de permanencia. Esta tasa, es dependiente de si el estudiante es el primer hijo en asistir a la universidad, siendo mayor en el primer año de estudios (Ishitani, 2003). Así mismo, los patrones intergeneracionales de la familia son determinantes en la adaptación del estudiante (Gold, 1995).

Sin duda, el factor financiero ha sido analizado como un factor que explica la permanencia en la universidad (Ishitani y DesJardins, 2002). Diferentes tipos de ayuda financiera afectan de manera distinta la deserción, mientras la subvención no favorece la permanencia del estudiante, el préstamo se asocia positivamente, debido al compromiso de pago futuro adquirido. Este factor se espera que explique la deserción estudiantil en las carreras estudiadas del presente estudio, debido a mayoritariamente los estudiantes provienes de familias de bajos ingresos.

Otras investigaciones, han incorporado factores explicativos relacionados con las condiciones socioeconómicas y el desempeño académico de los estudiantes encontrando, por ejemplo, que los estudiantes con menores ingresos al momento de iniciar sus estudios tienen mayores probabilidades de desertar (Díaz, 1999) y, que la retención de alumnos con padres de menor nivel de educación es muy baja, así como el rendimiento es menor para estudiantes que trabajan y disminuye con el número de horas trabajadas (Porto y Di Gresia, 2001).

Con el fin de incluir la dimensión dinámica al análisis, algunos autores han trabajado con modelos que permiten comparar la probabilidad de abandonar los estudios en cada periodo, concluyendo, en general, que la probabilidad de desertar no es constante a lo largo del tiempo, como por ejemplo los modelos desarrollados por DesJardins (2001); Dagenais et al. (2001). Estos modelos que consideran la dimensión temporal, se han transformado en una importante herramienta para realizar predicciones de tasas de titulación y tasas de deserción estudiantil, así como para identificar las causas que contribuyen positiva o negativamente. Por ello, el presente estudio utiliza estas técnicas para describir cómo la probabilidad de desertar cambia en el tiempo, desde un origen bien definido hasta la ocurrencia del evento.

\section{METODOLOGÍA}

Para saber cuándo el estudiante puede presentar un mayor riesgo de desertar y cuál es la probabilidad de que un estudiante, con ciertas características, deserte en el semestre actual dado que no lo ha hecho hasta este momento, se utilizan los modelos de duración o análisis de duración. El dato analizado es el tiempo transcurrido hasta que el estudiante deserta, y es probable que al final del periodo de observación no todos los estudiantes desertaran.

Para el análisis de los datos históricos, es necesario evaluar el riesgo de deserción, el que se entiende como la probabilidad de que un estudiante deserte en un momento del tiempo. La supervivencia o retención del estudiante, corresponderá a la probabilidad de que el estudiante permanezca dado que no ha desertado hasta el último semestre observado.

Por una parte, considerando estos conceptos es posible realizar un análisis no paramétrico, el cual permite evaluar el riesgo de desertar y la supervivencia de los estudiantes en el tiempo. Por otra parte, para explorar en los factores que explican esta deserción, se realiza un análisis semi paramétrico donde se determinan qué variables participan de la deserción de los estudiantes. 


\section{Análisis no paramétrico}

Para la cuantificación del riesgo acumulado de la deserción estudiantil durante el periodo de estudio, se aplicó el método no paramétrico de la curva de supervivencia de Kaplan Meier y la función de riesgo (Función Hazard) (Lancaster, 1992).

El método de Kaplan Meier (Lancaster, 1992) se basa en que un estudiante permanece un semestre, si es que ha permanecido todos los días de éste. Luego, es posible calcular para cada semestre la proporción de eventos que se observan en ese semestre.

Si se aplica el concepto de probabilidad condicional, para permanecer tres semestres hay que permanecer dos y luego un más. Esto se expresa en término probabilístico, como la multiplicación de las probabilidades. Luego, la supervivencia o retención del estudiante $\left(S\left(t_{i}\right)\right)$ en el tiempo $t_{i}$ se calculará mediante la siguiente fórmula recursiva (Lancaster, 1992):

$S\left(t_{i}\right)=\frac{r_{i}-m_{i}}{r_{i}} S\left(t_{i-1}\right)$

Donde, para cada semestre $i$ la retención se calcula como la retención del semestre anterior $\left(S\left(t_{i-1}\right)\right)$ multiplicada por la tasa de retención en ese periodo. En el denominador se tiene el número de estudiantes en el tiempo $i\left(r_{i}\right)$ que continuaban en el semestre anterior $(i-1)$ (expuestos al riesgo) y en el numerador, a ese valor se le resta el número de estudiantes $(m)$ que presentan el evento en el semestre $i$. Estos valores evaluados por cada semestre, son graficados en el tiempo obteniendo una curva de supervivencia.

\section{Función de riesgo (Hazard Function)}

Corresponde a la probabilidad de que el periodo finalice y exista un paso de un estado a otro, en un intervalo $t+\Delta t$. La función de riesgo $h(t)$, se expresa como:

$h(t)=\lim _{\Delta t \rightarrow 0^{+}} \frac{P(t \leq T<t+\Delta t \mid T \geq t)}{\Delta t}=\frac{f(t)}{S(t)}$

\section{Donde}

$\mathrm{f}(\mathrm{t})$ : es la función de densidad de la duración

$\mathrm{S}(\mathrm{t})$ : es la función de supervivencia con valores entre 1 y 0.

En el caso estudiado en este trabajo, la función de riesgo especifica la probabilidad condicional instantánea de que un estudiante experimente el suceso, es decir deserte en el intervalo $[t+\Delta t]$, dado que no lo ha experimentado (ha sobrevivido) hasta el momento $t$. En una interpretación alternativa, la función expresa el riesgo de que la duración termine en ese mismo intervalo (Lancaster, 1992). En este sentido, si el estudiante permanece en la Universidad, la función de riesgo expresa la probabilidad de que dicha permanencia tenga una duración $t$. Estas funciones son graficadas para realizar el análisis de los datos a través del tiempo.

\section{Análisis Semi-paramétrico: Modelo de Cox de Riesgo Proporcional}

Para la estimación de las variables y parámetros que explican el riesgo de deserción estudiantil, se aplicó modelo de Cox de Riesgos Proporcionales. En este modelo los riesgos para dos conjuntos diferentes de valores de las covariantes, se conserva en la misma proporción a lo largo del tiempo; de ahí el nombre de modelo de riesgos proporcionales. 
Se trata de calcular la tasa de deserción como una función del tiempo y de los factores explicatorios. El método más utilizado para la evaluación de la supervivencia es el Modelo de Cox de Riesgos Proporcionales cuya estimación es a través de máximo verosimilitud (Green, 1998), en el que la tasa de deserción se calcula como:

$\lambda\left(t, x_{1}, x_{2}, \ldots, x_{p}\right)=\lambda_{0}(t) * e^{b_{1} * x_{1}+b_{2} * x_{2}+\ldots+b_{p} * x_{p}}$

Por tanto, la tasa de deserción es el producto de dos componentes, uno $\lambda_{0}(\mathrm{t})$ que depende del tiempo $y$ otro $e^{b^{*} x}$ que depende de las variables de pronóstico o covariantes y no depende del tiempo. $\lambda_{0}(t)$ representa la función de riesgo cuando todas las covariantes valen cero, o el riesgo basal cuando no tiene sentido que alguna de las variables valga cero.

El producto $b^{\star} x$ es el perfil de riesgo de los estudiantes, donde $b_{i}$ corresponde a los parámetros estimados, que representan el cambio proporcional esperado en el riesgo, respecto al cambio en la variable explicativa $X$. Si una de las variables de pronóstico fuera dicotómica, codificada como 0 cuando no está presente la característica de la variable y 1 cuando está presente la característica de la variable, manteniendo iguales el resto de covariantes, se puede calcular los valores del término $e^{b^{*} x}$. Este término corresponde al riesgo relativo de la variable $X$.

El modelo de Cox se basa en que a menudo no se conoce la forma de $\lambda_{0}(t)$ y además no es de interés primordial, ya que el verdadero objetivo es valorar la influencia de los factores de pronóstico en la supervivencia. Por ello en el modelo de Cox no se determina $\lambda_{0}(t)$.

En consecuencia, para este estudio se propone que el perfil de riesgo $b_{i}{ }^{*} X_{i}$, basándose en las categorías de las variables, es una función de la forma:

f(Individual, Académica, Socioeconómica,Institucional)

Se sensibilizó el ingreso y exclusión de las variables de manera iterativa, eliminando aquellas que no eran significativas estadísticamente, hasta lograr un modelo parsimonioso y significativo. Las variables de las categorías individual, académica, socioeconómica e institucional, se describen más adelante.

\section{Base de Datos}

Se seleccionaron tres carreras de ingeniería cuyos estudiantes ingresaron según el proceso de postulación a las universidades, acorde al puntaje obtenido en la PSU en el año 2004. Considerando la privacidad de los datos, y que el objetivo es explorar en las carreras de ingeniería y observar posibles diferencias entre carreras, se procedió a nominar tres programas seleccionados como: Ingeniería A, Ingeniería B e Ingeniería C. El criterio para seleccionar estas carreras se basa en que a través del tiempo se han observado significativas tasas de deserción semestrales. Se considera un estudiante desertor aquel que renuncia o queda eliminado de la carrera, según la reglamentación que lo rige. Por ello, resulta interesante conocer cuáles son los factores que generan estos abandonos basándose en las variables y modelos que aborda el estudio.

Los datos fueron obtenidos desde el Sistema de Admisión y Registro Académico (SIMBAD) de la Universidad. Para el año 2004 el número total de estudiantes que ingresaron en las carreras anteriormente señaladas fueron 267 estudiantes, correspondiendo a Ingeniería A 25,5\% ( $\mathrm{N}=68)$, Ingeniería B 38,9\% ( $N=104)$ e Ingeniería C 35,6\% ( $N=95)$.

Los programas computacionales utilizados para el ordenamiento de los datos y graficación fueron MS Access y MS Excel, respectivamente. Para las estimaciones no paramétricas y modelo de Cox se utilizó Limdep versión 7.0 (Green, 1998). 


\section{Descripción de variables}

El criterio de selección de las variables en este trabajo fue acorde a la disponibilidad de ellos y los antecedentes reportados por la literatura. Desde aquí, se seleccionaron 30 variables subdivididas en cuatro categorías: individuales, académicas, socioeconómicas e institucionales. Dentro de la categoría individual se encuentran: género, estado civil, número de personas que viven con el estudiante, cercanía a la universidad, región de origen, edad de egreso del estudiante desde la enseñanza media y edad de ingreso a la universidad. En cuanto a la categoría académica se consideró: tiempo de permanencia en la carrera, condición de estado (desertor o no desertor), año de egreso de la enseñanza media, rama educacional, grupo de dependencia financiera del establecimiento, promedio de la PSU, promedio de notas de enseñanza media, preferencia de postulación a la carrera, lugar de la lista de selección, créditos inscritos totales al semestre, créditos aprobados totales al semestre, créditos reprobados totales al semestre, promedio ponderado acumulado al semestre y suspensión de estudios. La categoría socioeconómica consideró: situación laboral, horario de trabajo, horas de trabajo, participación en el financiamiento de sus estudios, nivel educacional del padre, nivel educacional de la madre e ingreso mensual bruto familiar. Este último, constituye el ingreso descontando los impuestos, previsión y salud. Respecto a la categoría institucional, se consideró: si el estudiante había obtenido en algún semestre becas, crédito universitario o ambos. En el Anexo 1, se encuentra el detalle de las variables y su abreviación.

Las variables relacionadas con las categorías individual y socioeconómica se obtuvieron desde la base de datos del DEMRE. Las variables de la categoría académica se obtuvieron desde el sistema SIMBAD y DEMRE; y las institucionales desde el sistema SIMBAD.

\section{Caracterización general de los estudiantes}

De los 267 estudiantes considerados, el $28,8 \%$ son mujeres y $100 \%$ son solteros, el $55,8 \%$ vive a no más de 40 kilómetros de distancia de la Universidad, y el 75,3\% vive en la octava región. La edad de ingreso promedio a la universidad fue de 20,1 años, mientras que la edad de egreso desde la enseñanza media de los estudiantes fue de 18,1 años. En cuanto a las características académicas $67,0 \%$ de los estudiantes desertó durante el periodo de análisis, siendo el tiempo promedio de permanencia de estos estudiantes de 4,2 semestres. Del total de estudiantes el $61,4 \%$ egresó el año anterior (2003) y el $57,7 \%$ proviene de colegios con financiamiento estatal. El puntaje promedio de la PSU fue de 537. De las características socioeconómicas, se puede observar que sólo el 1,0\% trabaja en forma regular, sin embargo, el $55,4 \%$ de los estudiantes dice que participa en el financiamiento de sus estudios y $60,3 \%$ de los estudiantes señala que en el hogar se tiene un ingreso bruto familiar mensual de bajo nivel de ingreso. En relación a la educación de los padres el $6,4 \%$ de los estudiantes posee padre y madre con una educación básica completa, el 33,0\% posee padres con una educación media completa, el $9,4 \%$ una técnica completa y el $11,6 \%$ tiene padres con enseñanza universitaria completa. Las variables relacionadas con la categoría institucional muestran que un $59,6 \%$ de los estudiantes recibió becas para financiamiento de un porcentaje del arancel anual durante el periodo de estudio, y el $48,3 \%$ recibió crédito universitario, es decir un estudiante puede obtener una beca si posee crédito universitario.

\section{RESULTADOS Y DISCUSIÓN}

\section{Estimaciones no-paramétricas de la deserción estudiantil}

Según los resultados de la supervivencia y función de riesgo, para los estudiantes de las carreras de ingeniería analizadas, tienen mayor probabilidad de supervivencia en el primer semestre, y luego disminuye a tasas decrecientes hasta el último semestre (ver figura 1). Es importante destacar que la supervivencia entre el primer y cuarto semestre disminuye con igual tendencia, la cual cambia a partir del quinto semestre. Sin embargo, es evidente que se muestra una deserción importante en los primeros tres semestres de las carreras de ingeniería. 


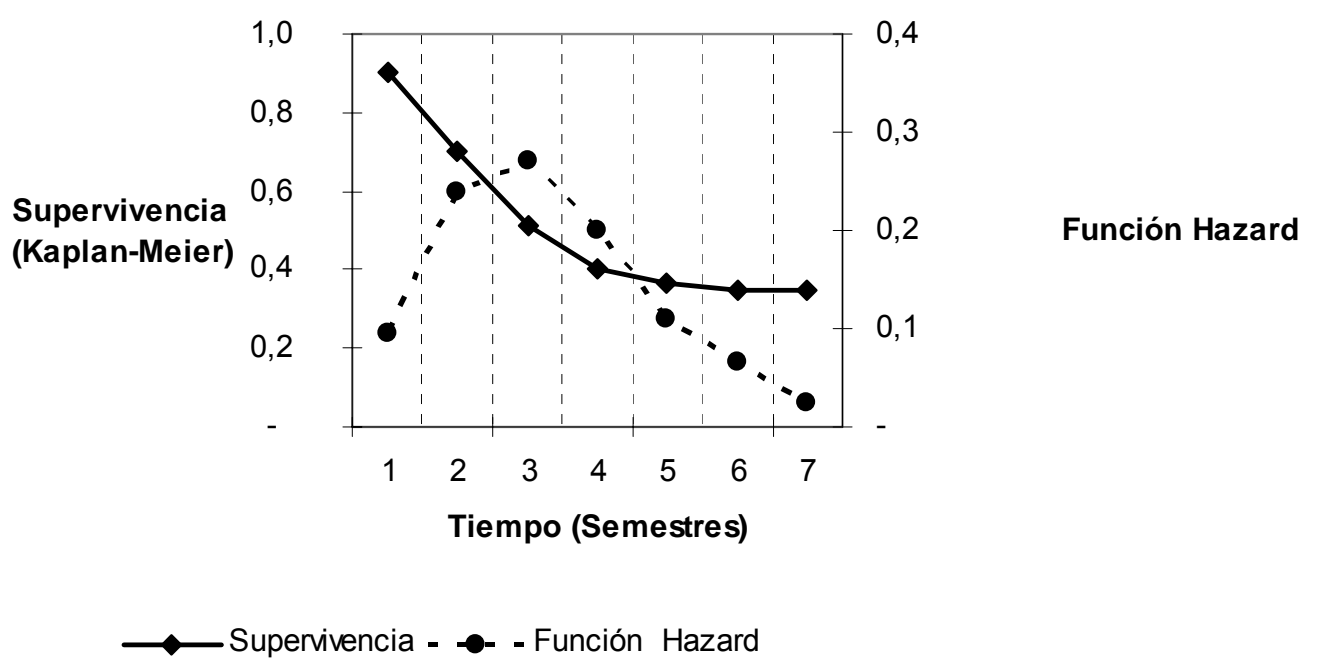

Fig. 1: Supervivencia (Kaplan-Meier) y Función Hazard de los estudiantes de ingeniería, cohorte 2004.

El riesgo de desertar (Función Hazard) que poseen los estudiantes de ingeniería, según la figura anterior, es mayor entre el segundo y cuarto semestre, alcanzando un valor máximo igual a $27 \%$ en el tercer semestre. A contar de este semestre el riesgo de desertar diminuye en forma sostenida, llegando a $3 \%$ en el último semestre. Esto indica que las mayores probabilidades de que el estudiante abandone la carrera, es en el primer y segundo año. Por ello, las acciones que permitan modificar las condiciones que no favorecen a la retención, deben ser abordadas durante este periodo.

Cabe señalar que la disminución del riesgo de deserción desde el tercer al séptimo semestre es de un $24 \%$, y el aumento desde el primer al tercer semestre es $17 \%$. Esto indicaría que los estudiantes aumentan el riesgo de deserción hasta el tercer semestre y luego disminuye en forma sostenida.

Al analizar cada carrera de manera individual, es posible evaluar la supervivencia y la función de riesgo. Para los estudiantes de ingeniería $A$, según la figura 2, la probabilidad de supervivencia muestra que ésta es mayor en el primer semestre, disminuyendo a tasas decrecientes hasta el quinto semestre alcanzado un valor de 39\%; este valor se mantiene constante hasta el séptimo semestre.

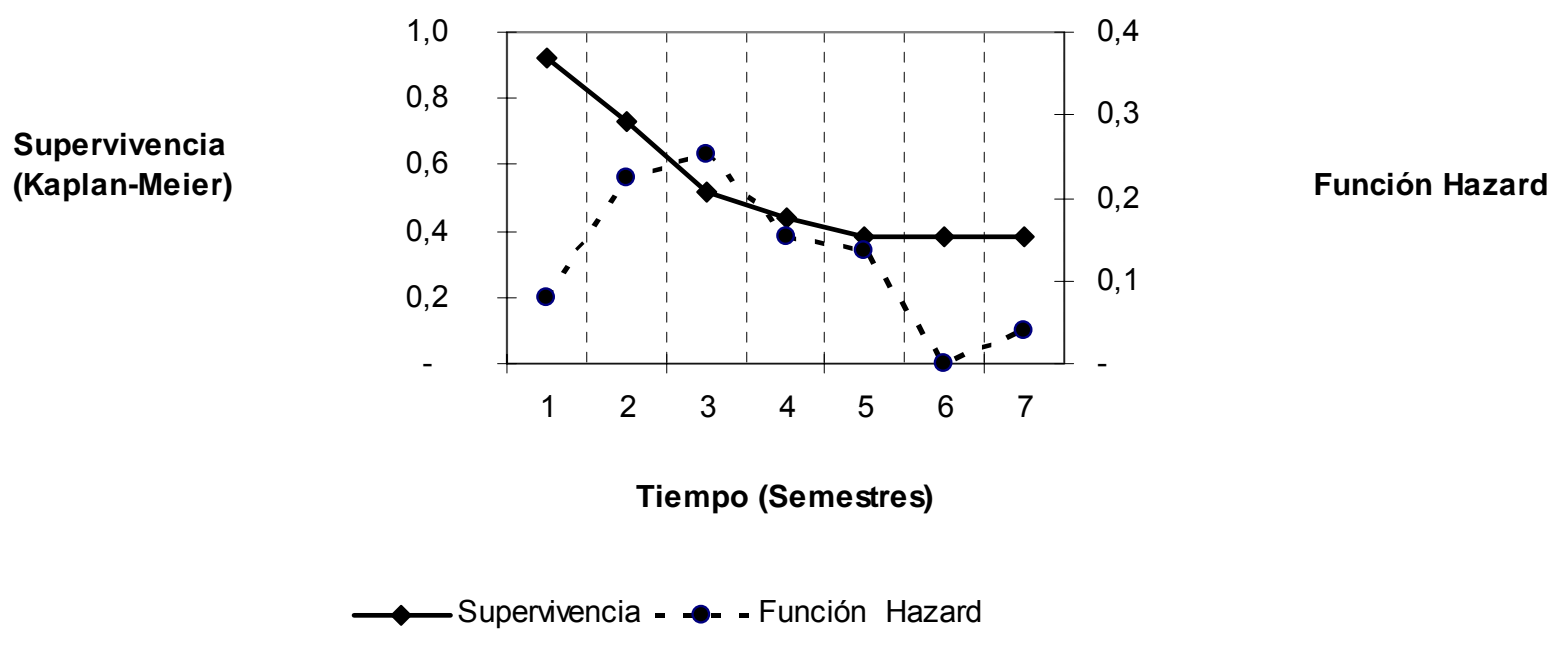

Fig. 2: Supervivencia (Kaplan-Meier) y Función Hazard de los estudiantes de ingeniería A, cohorte 2004. 
La Función Hazard en el primer semestre alcanza un valor igual a un $8 \%$, y luego aumenta a $23 \%$ en el segundo semestre, alcanzando su valor más alto de $25 \%$ en el tercer semestre. Luego el riesgo disminuye hasta llegar a cero en el sexto semestre, para volver a subir a un $4 \%$ en el séptimo semestre. Es importante destacar que en esta carrera el riesgo de deserción experimenta una disminución importante de un $25 \%$ entre el tercer y sexto semestre, sin embargo, sube al séptimo semestre.

Los estudiantes de ingeniería B poseen una alta probabilidad de supervivencia en el primer semestre, según lo muestra la figura 3, la cual disminuye al segundo semestre llegando a un $74 \%$ y continúa bajando con igual tendencia hasta el cuarto semestre, disminuyendo así en más de un $50 \%$ desde el primer al cuarto semestre. A partir del cuarto semestre en adelante, disminuye levemente hasta el último periodo de estudio, disminuyendo en un $5 \%$ desde el periodo cuarto al séptimo.

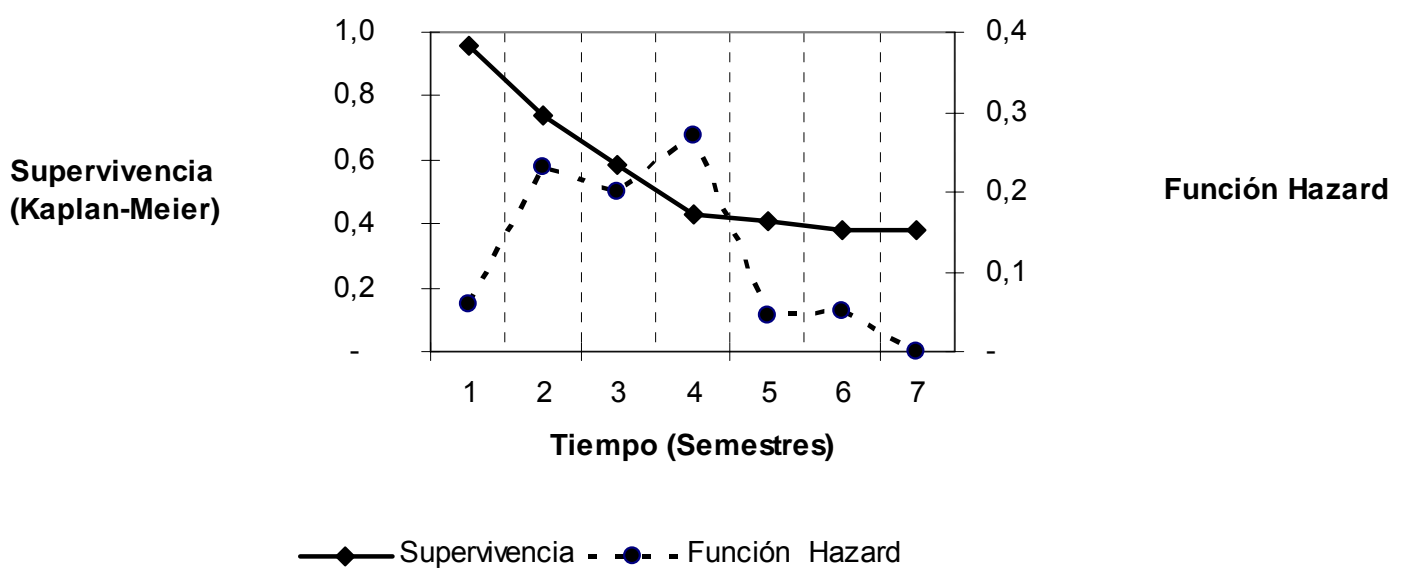

Fig. 3: Supervivencia (Kaplan-Meier) y Función Hazard de los estudiantes de ingeniería B, cohorte 2004.

Por su parte, el riesgo de deserción en el primer semestre alcanza un valor cercano a un $6 \%$ y luego sube en $17 \%$, llegando a un $23 \%$. Desde el segundo a tercer semestre disminuye en un $3 \%$ y para posteriormente subir a su valor más alto en el cuarto semestre a $27 \%$, y luego bajar en un $22 \%$ al quinto semestre, subir levemente en el sexto semestre y bajar a cero en el séptimo semestre. Es importante señalar que la función Hazard presenta tres aumentos y tres disminuciones en el mismo periodo de estudio, lo que indica que los estudiantes tardan aproximadamente seis semestres en estabilizar la probabilidad de deserción.

La supervivencia de los estudiantes de ingeniería C en el primer semestre alcanza $88 \%$, según lo muestra la figura 4, la cual disminuye hasta el tercer semestre con similar tendencia llegando a un $42 \%$, y continúa bajando desde el tercer semestre hasta el sexto semestre pero con una tendencia más leve, alcanzando un $27 \%$, el cual se mantiene constante en el séptimo semestre. Entre el primer y tercer semestre la supervivencia disminuye en un $46 \%$ y desde el tercer al sexto semestre disminuye en un $16 \%$. Esto indicaría que la probabilidad de deserción aumenta en los tres primeros semestres.

Los estudiantes de ingeniería $\mathrm{C}$ tienen un menor riesgo de deserción en el primer semestre igual a $14 \%$, el cual aumenta a $27 \%$ en el segundo semestre, alcanzando su valor máximo en el tercer semestre en un $36 \%$. Desde este semestre disminuye en un $20 \%$ al cuarto semestre y vuelve a subir del quinto al sexto alcanzando un valor de $15 \%$, para bajar a un $1 \%$ en el séptimo semestre.

Al comparar las tres carreras, se observa que los estudiantes de la ingeniería C presenta mayor riesgo de deserción en el tercer semestre (36\%), lo que podría estar asociado al género, ya que esta carrera tiene el mayor porcentaje de participación de mujeres en la matrícula $(76,8 \%)$, respecto a la 
ingeniería A que posee un 65,8\%. También es importante señalar que, los estudiantes de la ingeniería $\mathrm{C}$ posee en promedio una preferencia menor por la carrera respecto a los estudiantes de las otras carreras. Adicionalmente, esta diferencia en el comportamiento de la función de riesgo puede ser causada porque los estudiantes de la ingeniería A tiene una menor participación en el financiamiento de sus estudios (42\%), respecto a los estudiantes de la ingeniería A y B con 55 y $82 \%$, respectivamente.

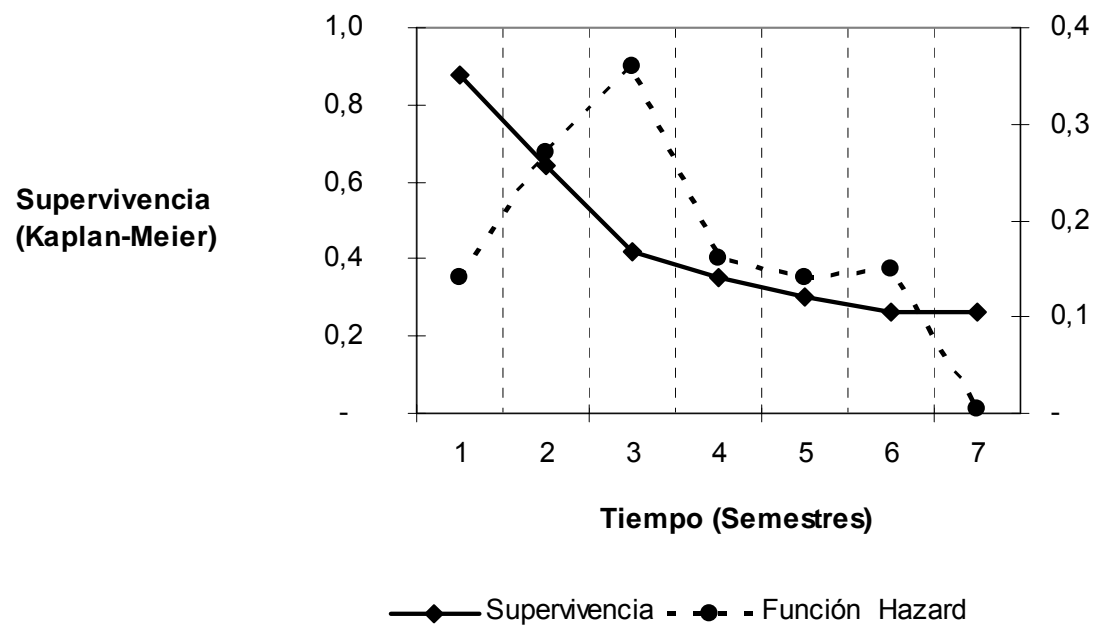

Función Hazard

Fig. 4: Supervivencia (Kaplan-Meier) y Función Hazard de los estudiantes de ingeniería C, cohorte 2004.

\section{Factores determinantes de la deserción estudiantil}

Para evaluar los factores que explican el fenómeno de deserción en los estudiantes de ingeniería, se realizaron estimaciones semi-paramétricas. Se presentan dos modelos en la tabla 1 , en el modelo 1 se observan que los coeficientes estimados son significativos, identificando a la variable BECA como un factor de riesgo, debido a su valor de riesgo relativo mayor que uno. Es decir, si el estudiante de ingeniería posee beca, esto no garantiza que éste no deserte, debido a que estos estudiantes han optado por desertar de la carrera. Lo anterior, puede deberse a que aquellos estudiantes que poseen becas, presentan menor compromiso en mantenerse, por tener menor endeudamiento, además son escasas las redes sociales que poseen al interior de la universidad, generalmente se trata de la primera generación que ingresa a la universidad y no encuentran a sus pares en educación en el entorno cercano, y por último se suma que a pesar de tener beca de financiamiento, el ingreso familiar no es suficiente.

Al igual que la variable anterior, la preferencia de postulación (PREF) muestra un valor de riesgo relativo que indicaría que mientras mayor es el valor de esta variable, y por ende menor preferencia a la carrera, existe mayor probabilidad de desertar de la carrera, si las demás variables se mantienen constantes. Según Cabrera et al., (1993) esto se asocia con la desmotivación por la carrera y en algunos casos por la universidad, dado que se presenta una barrera en la integración social de los estudiantes en los primeros meses de estudio universitario, por haberse matriculado en una carrera e institución que no era lo que el estudiante deseaba como primera prioridad. Estos resultados son concordantes con los obtenidos por Pascarella y Chapman (1983), quienes encontraron que la baja selectividad de los estudiantes al matricularse en una carrera aumenta el riesgo de deserción durante los primeros años de estudio, respecto de aquellos estudiantes que se matriculan en carreras e instituciones que tiene como primera prioridad.

Son factores de protección de la deserción, que el estudiante posea crédito universitario (CREDU), y si proviene desde un establecimiento de enseñanza media de formación científico humanista (RAMA). Además, es menos probable que el estudiante deserte si posee un buen rendimiento académico, reflejado a mayor valor del promedio ponderado acumulado (PPA). Esto último 
concuerda con los resultados obtenidos por Ishitani y Zinder (2006), quienes determinaron que el promedio de notas es un predictor de la obtención del título universitario. Específicamente, encuentra que el aumento de un punto en el promedio de notas aumenta más del doble la probabilidad que tiene un estudiante termine sus estudios; sin embargo, este efecto disminuye a medida que pasa el tiempo.

El factor puntaje promedio de la prueba de selección universitaria (PSUMED), posee un riesgo relativo cercano a 1, lo que indicaría que un aumento o disminución en un punto de este factor, no genera un cambio significativo en el riesgo de deserción de los estudiantes de ingeniería; sin embargo, corresponde a un factor de protección.

Tabla 1: Modelo de Cox de Riesgos Proporcionales para la deserción de estudiantes de ingeniería, admisión $2004\left({ }^{a}: *=\right.$ validez a un $10 \%$ de error; ${ }^{* *}=$ validez a un $5 \%$ de error; $* * *=$ validez a un $1 \%$ de error).

\begin{tabular}{|c|c|c|c|c|c|c|}
\hline \multirow{2}{*}{ Variable } & \multicolumn{3}{|c|}{ Modelo 1} & \multicolumn{3}{|c|}{ Modelo 2} \\
\hline & $\beta_{\mathrm{i}}{ }^{a}$ & St. Err. & $\mathbf{e}^{\beta}$ & $\beta_{\mathrm{i}}$ & St. Err. & $\mathbf{e}^{\beta}$ \\
\hline PPA & $-1,267^{* * *}$ & 0,121 & 0,282 & & & \\
\hline CREDU & $-1,073$ *** & 0,201 & 0,342 & $-1,224 * * *$ & 0,195 & 0,294 \\
\hline BECA & $0,567^{* * *}$ & 0,188 & 1,763 & 0,386 ** & 0,195 & 1,47 \\
\hline RAMA & $-0,342$ & 0,195 & 0,710 & & & \\
\hline PSUMED & $-0,003$ & 0,002 & 0,997 & $-0,005^{* * *}$ & $-0,002$ & 0,995 \\
\hline PREF & 0,062 & 0,050 & 1,06 & & & \\
\hline $\begin{array}{l}\text { INGFAM } \\
\text { EDADE }\end{array}$ & & & & $\begin{array}{r}-0,0007 * * \\
0,429 * * \\
\end{array}$ & $\begin{array}{c}-0,0003 \\
0,163 \\
\end{array}$ & $\begin{array}{r}0,999 \\
1,54 \\
\end{array}$ \\
\hline $\mathbf{N}$ & & & 261 & & & 225 \\
\hline$p$-valor & & & 0,000 & & & 0,000 \\
\hline
\end{tabular}

Para aislar el efecto de la variable rendimiento académico se excluyó del análisis la variable PPA, obteniendo un nuevo modelo, y así observar otros factores determinantes de la deserción (ver modelo 2). En el modelo 2, se presentan como variables de protección CREDU, PSUMED e engreso familiar (INGFAM). Estas dos últimas variables poseen un riesgo relativo cercano a 1, lo que indicaría que un aumento o disminución en un punto de este factor, no genera un cambio significativo en el riesgo de deserción de los estudiantes. Sin embargo, considerando que es un valor menor a 1 , correspondería a una variable de protección pero de un bajo impacto sobre el riesgo de deserción. Como factor de riesgo, al igual que en el modelo 1, es determinante la variable BECA, incorporándose la edad de egreso de los estudiantes enseñanza media (EDADE). Este, indicaría que a mayor edad de egreso desde la enseñanza media, mayor es la probabilidad de desertar del estudiante en ingeniería.

Los resultados obtenidos del modelo 2, son concordantes con los estudios realizados por Pascarella y Chapman (1983) quienes determinaron que el factor que explica en mayor porcentaje la deserción y el avance en la universidad es la preparación de la educación secundaria, la que se ve reflejada en los puntajes que los estudiantes alcanzan en las pruebas de ingresos a las universidades. Por su parte, la determinación del ingreso familiar como variable de protección, también concuerda con los resultados obtenidos por Díaz (1999), el cual determinó que mientras más alto es el ingreso familiar mayor es el tiempo que el estudiante permanecerá en la universidad antes de completar sus estudios y menor es el tiempo antes de abandonar sus estudios. Esto indica que los estudiantes que cuentan con menores ingresos al iniciar sus estudios tienen mayores probabilidades de desertar, mientras que los alumnos con mayores ingresos tienen mayores probabilidades de completar sus estudios.

\section{CONCLUSIONES}

Los estudiantes de ingeniería, presentan altos riesgos de deserción entre el primer y tercer semestre, siendo máximo en este último semestre, para luego descender a tasa creciente. Específicamente, en la ingeniería A se presenta una conducta similar para la supervivencia y riesgo de deserción, 
observándose un incremento al séptimo semestre. Los estudiantes de ingeniería $B$, presentan un comportamiento distinto en el riesgo entre el segundo y tercer semestre, y alcanzan el mayor riesgo en el cuarto semestre. Los estudiantes de ingeniería C, muestran el mayor riesgo de deserción en el tercer semestre para luego bajar en el cuarto semestre a niveles similares a la ingeniería $A$.

En ingeniería son factores de protección a la deserción el promedio ponderado acumulado (PPA), que posea el estudiante crédito universitario (CREDU), que provenga de un establecimiento educacional científico-humanista (RAMA), a mayor puntaje de ingreso en la prueba de selección universitaria (PSUMED) y el ingreso familiar (INGFAM). Esto último, indicaría la posibilidad de que a mayor ingreso familiar, existen más posibilidades de acceder a recursos, de que el estudiante no trabaje y por lo tanto disponga de más tiempo para sus estudios, logrando así mejores resultados académicos.

Son factores de riesgo que el estudiante posea beca (BECA), a mayor lugar en la preferencia de postulación (PREF) y a mayor edad de egreso de enseñanza media (EDADE). Si bien la BECA aparece como un factor de riesgo, esto podría deberse a que aquellos estudiantes que las poseen, presentan condiciones que dificultan su integración social debido a su escasa red social, además, los ingreso familiares no son suficientes para cubrir todos los costos universitarios, y si a esto se suma el bajo compromiso con metas y logros académicos producto de ingresar a una carrera que no era de su primera prioridad, la probabilidad de deserción aumenta.

La evaluación no paramétrica de la deserción, permite hacer una descripción sobre la supervivencia de los estudiantes en el tiempo, entregando el riesgo de desertar en el tiempo. Esto, permite considerar acciones que involucren la disminución del riesgo de deserción, en temporalidades pertinentes, según los resultados del análisis.

La aplicación del modelo semi-paramétrico, permitió identificar factores que explicarían las motivaciones de desertar en una carrera de ingeniería o de aquellos factores que favorecen la retención de los estudiantes. Esto, demuestra la potencialidad del modelo, al revelar ciertas características en las cuales se pueden motivar, en el caso de las variables protectoras, o bien desincentivar o modificar, en el caso de los factores de riesgo. Además de identificar los factores, es posible estimar el riesgo de deserción por cada factor identificado, pudiendo detenerse en aquellas que generan efectos importantes en la deserción o retención. De esta manera, se pueden generar mecanismos que permitan atender estos factores y mejorar la retención de los estudiantes de ingeniería.

Finalmente, de un conjunto de estudiantes de ingeniería que ingresen a estudiar en primer año, estos modelos permiten identificar en cada estudiante, su potencial riesgo de deserción, con lo cual es posible implementar programas de trabajo con aquellos estudiantes con alto riesgo de manera de disminuir la probabilidad que el estudiante no continúe sus estudios de ingeniería en la universidad.

\section{AGRADECIMIENTOS}

A la Dirección de Investigación de la Universidad Católica de la Santísima Concepción, por el financiamiento parcial y hacer posible su publicación. A Catterina Sobenes de la Universidad quien entregó sugerencias y aportes en la revisión de este documento.

\section{REFERENCIAS}

Bean, J.P.; Interaction effect based on class level in an exploratory model of college student dropout syndrome. American Educational Research Journal: 22(1), 35-64, (1985).

Bean, J.P. y S. Eaton; The psychology underlying successful retention practices. Journal of College Student Retention Research, Theory \& Practice: 3(1), 73-89, (2001). http://baywood.metapress.com/app/home/contribution.asp?referrer=parent\&backto=issue,6,7;journal, 28,36; searcharticlesresults, 1,7;. Acceso: 25 de octubre de 2008. 
Boudon, R.; La desigualdad de oportunidades. La movilidad social en las sociedades industriales. Ed. Lania, Barcelona, España (1983)

Bourdieu, P.; La distincion. Crítica social del juicio. $2^{a}$ ed. Altea, Taurus-Alfaguara, Madrid, España (1984).

Braxton, J., M. Duster y E. Pascarella; Causal modeling and path analysis: An introduction and an illustration in student attrition research. Journal of College Student Development: 29(3), 263-272 (1988).

Cabrera, A., A. Nora y M. Castañeda; College Persistence: Structural Equations Modelling Test of Integrated Model of Student Retention. Journal of Higher Education: 64(2), 123-320 (1993). http://www.jstor.org/pss/2960026. Acceso: 25 de octubre de 2008.

Canales, A. y D. De los Ríos; Factores explicativos de la deserción universitaria. Revista Calidad en la Educación, Ministerio de Educación de Chile: 26, 173-201 (2007).

Dagenais, M., C. Montmarquette y N. Viennot-Briot; Dropout, School Performance and Working while in school: An econometric model with heterogeneous groups. Scientific Series, CIRANO, Montreal, Canada: 63, pp. 25 (2001).

DesJardins, S.; A Comment on Interpreting Odds-Ratios When Logistic Regression Coefficients are Negative. Association for Institutional Research Professional File: 81, 1-10 (2001). http://www.airwe b.org/page. asp?page=73\&apppage=69. Acceso: 25 de octubre de 2008.

Díaz, M.D.; Extended Stay at University: An Application of Multinomial Logit and Duration Models. Journal of Applied Economics: 31(11), 1411-1422 (1999). http://www.ingentaconnect.com/content/ routledg/raef/1999/00000031/00000011/art00013. Acceso: 25 de octubre de 2008.

Díaz, Ch.; Modelo conceptual para la deserción estudiantil universitaria chilena. Revista Estudios Pedagógicos, Universidad Austral de Chile: 34(2) (2008). En prensa.

Donoso, S. y E. Schiefelbein; Análisis de los modelos explicativos de retención de estudiantes en la universidad: una visión desde la desigualdad social. Revista Estudios Pedagógicos Universidad Austral de Chile: 33(1), 7-17 (2007).

Gold, J.; An Intergenerational Approach to Student Retention. Journal of College Student Development: 36(2), 182-187 (1995).

González, L. y D. Uribe; Estimaciones sobre la repitencia y deserción en la educación superior chilena. Consideraciones sobre sus implicancias. Revista de la Calidad de la Educación: 17, 75-90 (2002)

González, L.E.; Estudio sobre la repitencia y deserción en la educación superior chilena. Digital Observatory for higher education in Latin America and The Caribbean. IESALC-UNESCO, SantiagoChile (2005).

Green, W.; Análisis econométrico. Tercera edición. Ed. Prentice Hall, pp. 911, Madrid-España (1998).

INIDICES, Indicadores, Números y Datos sobre Instituciones y Carreras de Educación Superior, Superior de Educación, Ministerio de Educación de Chile (2006).

Ishitani, T.; A longitudinal approach to assessing attrition behavior among first-generation students: Time-varying effects of pre-college characteristics. Research in Higher Education: 44(4), 433-449 (2003). http://www.springerlink.com/content/w38702536I83j612/. Acceso: 25 de octubre de 2008. 
Ishitani, T. y S. DesJardins; A longitudinal investigation of dropout from college in the United States. Journal of College Student Retention: 4(2), 173-201 (2002).

Ishitani, T. y T. Zinder; Longitudinal Effect of Collage Preparation on College Retention. Association for Institutional Research: 9, pp. 16 (2006).

Kuh, G.; Organizational culture and student persistence: prospects and puzzles. Journal of College Student Retention: 3(1), 23-39 (2002).

Lancaster, T.; The econometric analysis of transition data. Brown University. Cambrige University Press, pp. 364 (1992).

Ley 18.962, Ley Orgánica Constitucional de Enseñanza. Ministerio de Educación de Chile (1990).

Ministerio de Educación de Chile; Compendio Estadístico, estadísticas institucionales (en línea), (2007), http://compendio.educasup.cl/comp_esta_inst.html. Acceso: 9 de noviembre de 2008.

Pascarella, E. y D. Chapman; A multi-institutional, path analytic validation of Tinto's model of college withdrawal. American Educational Research Journal: 20(1), 87-102 (1983).

Pascarella, E. y P. Terenzini; Predicting voluntary freshman year persistence / withdrawal behavior in a residential university: A path analytic validation of Tinto's model. Journal of Educational Psychology: 75(2), 215-266 (1983).

Porto, A. y L. Di Gresia; Rendimiento de estudiantes universitarios y sus determinantes. Asociación de Argentina de Economía Política, pp. 24, Buenos Aires-Argentina (2001).

Prieto, A.; Indecisión vocacional: pérdidas y "perdidos" en la educación superior. Revista de la Calidad de la Educación: 17, 145-163 (2002).

Spady, W.; Dropouts from higher education: toward an empirical model. Interchange: 19(2), 38-62 (1971).

St. John, E., A. Cabrera, A. Nora y E. Asker; Economic influences on persistence. J.M. Braxton. Reworking the student departure puzzle: New theory and research on college student retention. Nashville: Vanderbilt University Press, USA, 29-47 (2000).

Tinto, V.; Dropout From Higher Education: A Theoretical Synthesis of Recent Research. Journal of Higher Education: 45(1), 89-125. (1975).

Tinto, V.; Definir la deserción: una cuestión de perspectiva. ANUIES, México, Revista de Educación Superior: 71, 33-51. (1989).

UNESCO, Organización de las Naciones Unidas para la Educación, la Ciencia y la Cultura; Repetition at high cost in Latin America and the Caribbean. IESALC-UNESCO, (2004). 
Anexo 1

Descripción detallada de las variables operacionales incluidas en el modelo según categoría

Categoría: INDIVIDUAL

\begin{tabular}{|c|c|c|}
\hline Número & Nombre & Descripción \\
\hline 1 & GENERO & $\begin{array}{l}1=\text { Masculino } \\
0=\text { Femenino }\end{array}$ \\
\hline 2 & ECIVIL & $\begin{array}{l}\text { Estado Civil del estudiante al ingreso a la Universidad. } \\
1=\text { Soltero } \\
0=\text { en otro caso. }\end{array}$ \\
\hline 3 & GFAM & Número de personas que viven con el estudiante. \\
\hline 4 & CERCA & $\begin{array}{l}\text { Si el estudiante vive cerca del establecimiento } \\
1=\mathrm{SI} \\
0=\mathrm{NO}\end{array}$ \\
\hline 5 & REGION & Número de la región del país en la cual reside el estudiante. \\
\hline 6 & EDADE & Edad en años del estudiante de egreso de enseñanza media \\
\hline 7 & EDADI & Edad en años de ingreso del estudiante a la universidad \\
\hline
\end{tabular}

\begin{tabular}{|c|c|c|}
\hline \multicolumn{3}{|r|}{ Categoría: ACADÉMICA } \\
\hline Número & Nombre & Descripción \\
\hline 8 & TIEMPO & $\begin{array}{l}\text { Número de semestres de permanencia del estudiante en la } \\
\text { carrera. }\end{array}$ \\
\hline 9 & CENSU & $\begin{array}{l}\text { Indica si el estudiante es desertor o se encuentra censurado: } \\
1=\text { Si es desertor } \\
0=\text { Censurado (no se ha presentado el evento) }\end{array}$ \\
\hline 10 & ANOEG & $\begin{array}{l}\text { Corresponde al año en el cual el estudiante egresó de la } \\
\text { enseñanza media. }\end{array}$ \\
\hline 11 & RAMA & $\begin{array}{l}\text { Identifica si la modalidad o rama educacional de enseñanza } \\
\text { media es científico humanista: } \\
\begin{array}{l}1=\mathrm{SI} \\
0=\mathrm{NO}\end{array}\end{array}$ \\
\hline 12 & GDEPE & $\begin{array}{l}\text { Identifica la dependencia financiera del establecimiento } \\
1 \text { = Particular pagado (financiamiento privado) } \\
2 \text { = Particular Subvencionado (con financiamiento compartido) } \\
3 \text { = Con financiamiento del Estado }\end{array}$ \\
\hline 13 & PSUMED & Puntaje estándar promedio de la PSU \\
\hline 14 & NOTEM & Promedio de notas del estudiante en enseñanza media. \\
\hline 15 & PREF & $\begin{array}{l}\text { Número de orden de preferencia que marcó el código de la } \\
\text { carrera }\end{array}$ \\
\hline 16 & LUGAR & $\begin{array}{l}\text { Posición en la lista de selección a la carrera según puntaje } \\
\text { ponderado decreciente. }\end{array}$ \\
\hline 17 & CREDI & Número de créditos inscritos según el periodo lectivo. \\
\hline 18 & CREDA & Número de créditos aprobados según el periodo lectivo \\
\hline 19 & CREDR & Número de créditos reprobados según el periodo lectivo. \\
\hline 20 & PPA & $\begin{array}{l}\text { Promedio ponderado por créditos acumulados según el periodo } \\
\text { lectivo. }\end{array}$ \\
\hline 21 & SEMSUP & $\begin{array}{l}\text { Señala si el estudiante suspendió al menos un semestre de } \\
\text { estudio } \\
\begin{array}{l}1=\mathrm{SI} \\
0=\mathrm{NO}\end{array}\end{array}$ \\
\hline
\end{tabular}


Categoría: SOCIOECONOMICA

\begin{tabular}{|c|c|c|}
\hline Número & Nombre & Descripción \\
\hline 22 & WORK & $\begin{array}{l}\text { Indica si el estudiante trabaja: } \\
\begin{array}{l}1=\mathrm{SI} \\
0=\mathrm{NO}\end{array}\end{array}$ \\
\hline 23 & HWORK & $\begin{array}{l}\text { Horario de trabajo, donde: } \\
0=\text { no trabaja } \\
1=\text { diurno } \\
2=\text { Vespertino } \\
3=\text { Nocturno } \\
4=\text { sin horario fijo. }\end{array}$ \\
\hline 24 & HRWORK & Horas que trabaja a la semana. \\
\hline 25 & PARFIN & $\begin{array}{l}\text { El estudiante participa en el financiamiento de sus estudios: } \\
\begin{array}{l}1=\mathrm{SI} \\
0=\mathrm{NO}\end{array}\end{array}$ \\
\hline 26 & EDPAD & $\begin{array}{l}\text { Nivel educacional del padre del estudiante } \\
1=\text { Sin estudio } \\
2=\text { Básica incompleta } \\
3=\text { Básica completa } \\
4=\text { Media incompleta } \\
5=\text { Media completa } \\
6=\text { Centro de Formación Técnica incompleta } \\
7=\text { Centro de formación Técnica completa } \\
8=\text { Universitaria incompleta } \\
9=\text { Universitaria completa } \\
10=\text { Otros estudios }\end{array}$ \\
\hline 27 & (1) & $\begin{array}{l}\text { Nivel educacional de la madre del estudiante } \\
1=\text { Sin estudio } \\
2=\text { Básica incompleta } \\
3=\text { Básica completa } \\
4=\text { Media incompleta } \\
5=\text { Media completa } \\
6=\text { Centro de Formación Técnica incompleta } \\
7=\text { Centro de formación Técnica completa } \\
8=\text { Universitaria incompleta } \\
9=\text { Universitaria completa } \\
10=\text { Otros estudios }\end{array}$ \\
\hline 28 & INGFAM & Ingreso bruto mensual familiar \\
\hline
\end{tabular}

\section{Categoría: INSTITUCIONAL}

\begin{tabular}{l|ll}
\hline Número & Nombre & \multicolumn{1}{c}{ Descripción } \\
\hline $\mathbf{2 9}$ & BECA & El estudiante ha obtenido becas de la institución \\
& & SI $=1$ \\
& & NO $=0$ \\
30 & CREDU & El estudiante ha obtenido crédito universitario \\
& & SI = 1 \\
& & NO $=0$
\end{tabular}


